\title{
encaixAR: um livro de atividades em Realidade Aumentada para PNEE
}

\author{
Guilherme Augusto Ferreira ${ }^{1}$, Melise Maria V. de Paula ${ }^{1}$ \\ ${ }^{1}$ Instituto de Matemática e Computação - Universidade Federal de Itajubá (UNIFEI) \\ Caixa Postal: 50 - CEP: 37500-903 - Itajubá - MG - Brasil \\ \{guilhermeferreira, melise\}@unifei.edu.br
}

\begin{abstract}
This paper shows an AR (Augmented Reality) application on educational context applied to PSEN (Pupils with Special Educational Needs). The work describes the application and presents the result of the evaluation with PSEN. The application "encaixAR" simulates a book with virtual pages where each page presents a different task, involving the construction of words with missing letters and syllables. The obtained results demonstrate that AR is a technology that, when properly adapted, provides a satisfactory and motivating experience for PSEN.
\end{abstract}

Resumo. Este artigo apresenta uma aplicação de RA (Realidade Aumentada) no contexto educacional para PNEE (Portadores de Necessidades Educativas Especiais). O trabalho descreve uma aplicação desenvolvida (o encaixAR) e apresenta os resultados da avaliação com crianças PNEE. O encaixAR simula um livro com páginas virtuais onde cada página apresenta uma atividade diferente, envolvendo a construção de palavras com letras ou sílabas faltantes. Os resultados obtidos demonstram que a $R A$ é uma tecnologia que, quando devidamente adaptada, proporciona uma experiência satisfatória e motivante para PNEE.

\section{Introdução}

O uso da Realidade Aumentada (RA) no contexto educacional não é um evento inédito, porém, para PNEE (Portadores de Necessidades Educativas Especiais) existem recursos da RA que precisam ser adaptados visando uma interação efetiva e com o mínimo de obstáculos possíveis.

Segundo Bechtold (2005), o indivíduo PNEE é aquele que apresenta em caráter temporário ou permanente, significativas diferenças físicas, sensoriais ou intelectuais, decorrente de fatores inatos ou adquiridos. Essas características particulares acarretam dificuldades em sua interação com o meio social, necessitando de recursos especializados capazes de desenvolver seu potencial e superar ou minimizar suas dificuldades [Bechtold, 2005].

Os objetos de aprendizagem digitais desenvolvidos para esse público devem minimizar as dificuldades naturais impostas pela deficiência [Souza, 2010]. Existem vários objetos de aprendizado construídos em diversas tecnologias. A RA é uma delas. Segundo Kirner e Kirner (2008) "Realidade Aumentada (RA) é a inserção de objetos virtuais no ambiente físico, mostrada ao usuário, em tempo real, com o apoio de algum dispositivo tecnológico, usando a interface do ambiente real, adaptada para visualizar e manipular os objetos reais e virtuais." 
As características de RA aliadas ao processo educacional proporcionam um aprendizado rico e satisfatório ao aluno. Essas características são: interatividade, motivação (envolvimento), independência (autonomia), adequação e colaboração [Kirner, 2013]. Para PNEE, a característica de adequação pode ser considerada um fator determinante para o desenvolvimento de aplicações.

Este trabalho apresenta o encaixAR (uma aplicação desenvolvida em RA para PNEE) que foi elaborado a partir da evolução do trabalho de Paula, Ferreira e Silva (2012), onde os autores detalham (da concepção à validação) uma aplicação simples em RA para PNEE. Porém, os resultados deste trabalho apontaram que a aplicação não alcançou plena satisfação do público envolvido.

A aplicação de Paula, Ferreira e Silva (2012) apresentava poucos recursos de interação (exibe uma figura e um som quando um determinado marcador é exposto). Além disso, o conteúdo era muito simples para a maioria dos PNEE, o que fazia com que não se sentissem desafiados e, consequentemente, não demonstravam muito interesse em utilizar a aplicação novamente. Diante do exposto, o encaixAR surgiu com a proposta de um ambiente altamente interativo e com atividades condizentes com o nível dos estudantes.

O objetivo principal desse trabalho é apresentar uma aplicação com RA adaptada para PNEE. O trabalho avalia os seguintes fatores: usabilidade, legibilidade dos elementos virtuais, aprendibilidade, adequação das atividades e satisfação do usuário. Não faz parte do escopo desse trabalho avaliar o aprendizado.

Este trabalho está organizado como se segue: na Seção 2 são apresentados estudos relacionados. Na Seção 3 está descrito o software desenvolvido e na Seção 4 são detalhados experimento realizado e participantes envolvidos. A Seção 5 apresenta os resultados obtidos no processo de avaliação. E, por fim, a Seção 6 apresenta as conclusões.

\section{Revisão Bibliográfica}

Apesar de estar descrito na literatura um grande número de trabalhos que empregam RA no ensino das mais diversas áreas do conhecimento, segundo Vaghetti e Botelho (2010) é reduzido o número de trabalhos que tratam especificamente de educação para PNEE com RA.

Em Dainese e Gabin (2009) é apresentado o AMCARA (Ambiente de Comunicação Alternativo de Realidade Aumentada), que proporciona um conjunto de ambientes em RA desenvolvidos especialmente para pessoas com deficiências graves. Esse software torna vários ambientes acessíveis aos deficientes, sendo possível, por exemplo, acessar a web através de um mecanismo de comunicação adaptado.

Fernandes et al (2012) apresentam um software que auxilia o ensino do campo girante de um motor de corrente alternada. Em razão da dificuldade do aluno entender os fenômenos eletromagnéticos, foi criado um software em RA que simula virtualmente esse processo com recursos visuais. Deste modo, ao enxergar o comportamento desse sistema, o aluno consegue entendê-lo.

Em Kaufmann (2003), é apresentado o Construct3D, um software educativo que aborda o conteúdo pedagógico relacionado ao ensino de Geometria. O software oferece um conjunto de itens primitivos para a construção de imagens virtuais pelo usuário 
como pontos, linhas, planos, cubos, esferas, cilindros e cones. Além disso, permite ao usuário realizar operações de interseção, operações booleanas, operações de simetria e delimitação de medidas.

\section{Desenvolvimento}

O processo de desenvolvimento do encaixAR foi baseado na metodologia de desenvolvimento de software RUP considerando as seguintes etapas: concepção, elaboração, construção e validação [Piske, 2003].

Os aspectos analisados e os requisitos funcionais e não funcionais identificados nas etapas de concepção e elaboração estão detalhados em Paula, Ferreira e Silva (2012). Estes requisitos foram definidos em conjunto com uma instituição de ensino que atende crianças PNEE, onde professoras do ensino fundamental, que atuaram como colaboradoras, definiram as atividades a serem desenvolvidas na aplicação.

$\mathrm{Na}$ etapa de construção foram analisadas diferentes ferramentas de autoria buscando garantir que as características identificadas na etapa anterior pudessem ser atendidas. O resultado desta análise está apresentado na Seção 3.1. Além disso, foram realizados sucessivos testes para garantir uma versão estável do encaixAR para a validação. Foi necessário desenvolver um suporte físico para facilitar a interação dos PNEE com o encaixAR e reduzir as chances de erros decorrentes da utilização dos artefatos físicos que compõem o ambiente de RA definido.

$\mathrm{Na}$ validação foi realizado um experimento em uma escola que atende exclusivamente crianças PNEE. O objetivo principal foi analisar a adequação do encaixAR em relação ao contexto considerado: software educacional para PNEE.

\subsection{Ferramentas de autoria}

Segundo Souza, Moreira e Kirner (2012) existem dois meios para se construir uma aplicação em RA, ou seja, o desenvolvedor deve escolher entre Programação ou Ferramentas de autoria.

Uma ferramenta de autoria, segundo Santin (2008) coloca uma camada de abstração no desenvolvimento, ou seja, o desenvolvedor da ferramenta de autoria cria um paradigma para que outra pessoa (com um nível menor de conhecimento técnico) possa criar uma aplicação. Geralmente esse paradigma é de alto nível. O usuário configura as propriedades através de arquivos de texto e insere arquivos em diretórios pré-definidos pela ferramenta de autoria.

O desenvolvimento utilizando ferramentas de autoria exige pouco conhecimento técnico do desenvolvedor. Por outro lado, existem várias ferramentas de autoria, cada uma com objetivos e paradigmas diferentes. Com isso, o desenvolvedor pode estar limitado somente aos recursos disponibilizados pelo desenvolvedor da ferramenta [Souza, Moreira e Kirner, 2012].

A ferramenta de autoria escolhida para o desenvolvimento do encaixAR foi o basAR (Behavioral Authoring System for Augmented Reality). O basAR permite que os pontos de ação associados a um marcador tenham um comportamento dinâmico [Cerqueira e Kirner, 2011]. O paradigma utilizado para se desenvolver uma aplicação utilizando o basAR é baseado no conceito de autômatos. 
O fator que mais contribuiu para a escolha do basAR foi a flexibilidade para construir aplicações interativas. O basAR proporciona recursos para interagir com os objetos virtuais em tempo real de maneira estável, suporte a arquivos de áudio e animações com os objetos virtuais.

\section{2. $O$ encaix $A R$}

A estrutura do encaixAR pode ser entendida sob duas perspectivas: física e lógica. A Figura 1 mostra a estrutura física, composta por: câmera USB, suporte para câmera, base para página, barra limitadora e atuador.

Foi necessário construir uma infraestrutura que mantivesse a câmera fixa e o marcador de referência (impresso na página) sempre visível. Para isto, foi criado um artefato com canos PVC (Figura 1 - A e B), madeira e uma câmera USB. O item C da Figura 1 é responsável por posicionar os objetos 3D na tela do computador. Isso ocorre quando a câmera identifica o marcador presente no item $\mathrm{C}$ da Figura 1 . $\mathrm{O}$ marcador móvel (chamado atuador) foi fixado na ponta de uma tábua pequena e fina para permitir o acesso aos objetos 3D (Figura 1 - D). Uma barra foi utilizada para limitar a distância vertical entre o atuador e a folha (Figura 1 - E).

A câmera USB é conectada ao computador e o usuário interage com o encaixAR olhando somente para a tela. A Figura 3 mostra alguns screenshots em tempo de execução. Esses screenshots são exatamente a visão do encaixAR pelo usuário.

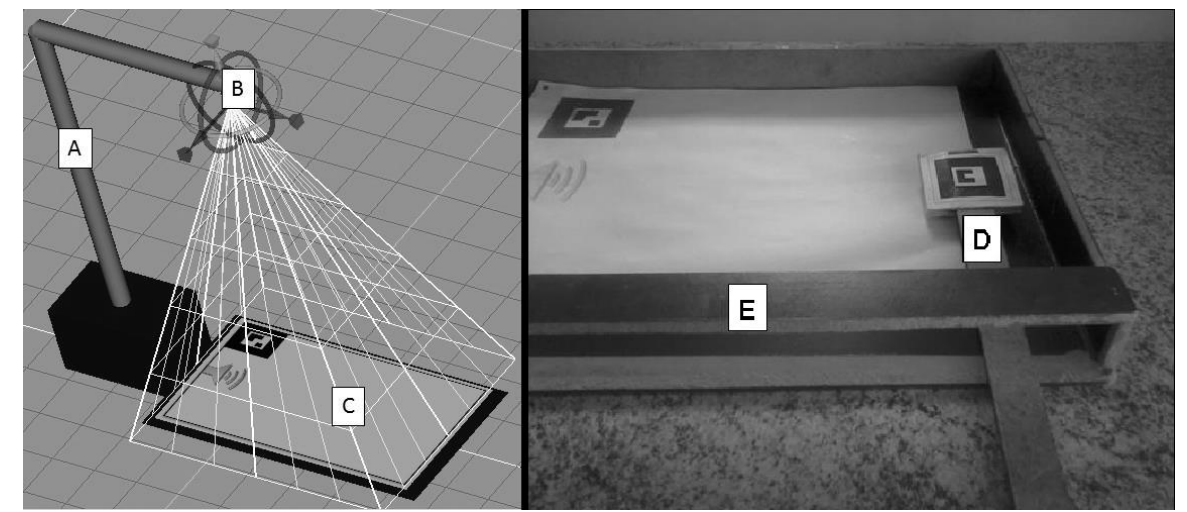

\section{Figura 1. Estrutura física do encaixAR - A) Suporte para câmera - B) Câmera USB - C) Base com a página física - D) Atuador - E) Barra limitadora.}

No que se refere à estrutura lógica, o encaixAR permite realizar dois tipos de atividades: marcar e arrastar. Quando a atividade é marcar, basta colocar o marcador atuador sobre o objeto para desencadear os eventos. Quando é arrastar, o usuário deve utilizar o atuador para conduzir manualmente o objeto a uma determinada posição.

O encaixAR simula um livro, porém com páginas virtuais. A cada página do livro, está associada a uma atividade diferente. Ao acertar uma atividade, o usuário tem a opção de seguir para a próxima atividade acionando um novo botão em formato de seta que aparece no canto da página. Por outro lado, ao errar, o usuário é estimulado a fazer uma nova tentativa.

O marcador de referência (Figura 2 - A) é responsável somente por posicionar os objetos 3D na página. A tecla de som (Figura 2 - B) emite uma instrução relacionada à respectiva atividade quando acionada pelo atuador. A tecla de passagem de página 
(Figura 2 - C) fica virtualmente oculta até que o usuário conclua a atividade proposta. Ao concluir a atividade, a seta se torna visível, e quando tocada troca a atividade. Esse esquema é ilustrado na Figura 2.

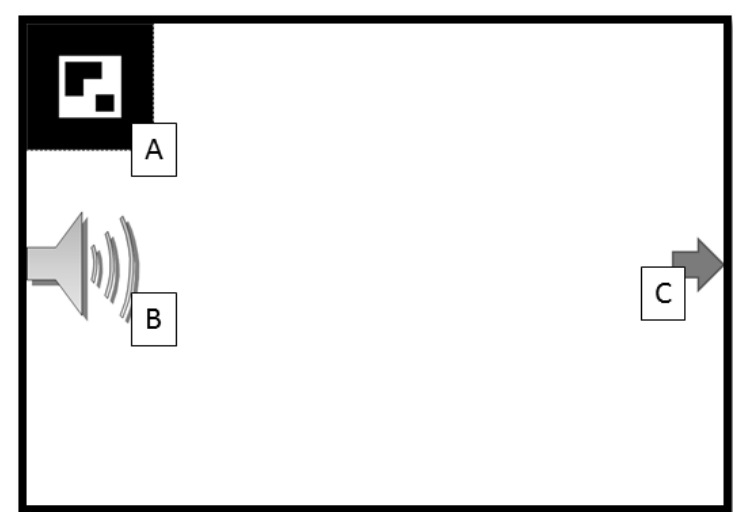

Figura 2. Página física do encaixAR.

A Figura 3 ilustra o encaixAR em funcionamento. Na primeira imagem, o encaixAR exibe um botão para iniciar as atividades. Quando esse botão é acionado, a primeira atividade surge imediatamente acompanhada pelo respectivo som (no caso do avião, reproduz o texto: "Marque a alternativa correta"). Quando o atuador é colocado sobre a alternativa correta, a letra vai automaticamente até o espaço correto e monta a palavra. Imediatamente após um acerto, o botão de troca de página se torna visível no ambiente virtual acompanhado de um som que sugere a possibilidade de troca de atividade. Caso o usuário acione, a próxima atividade aparece.

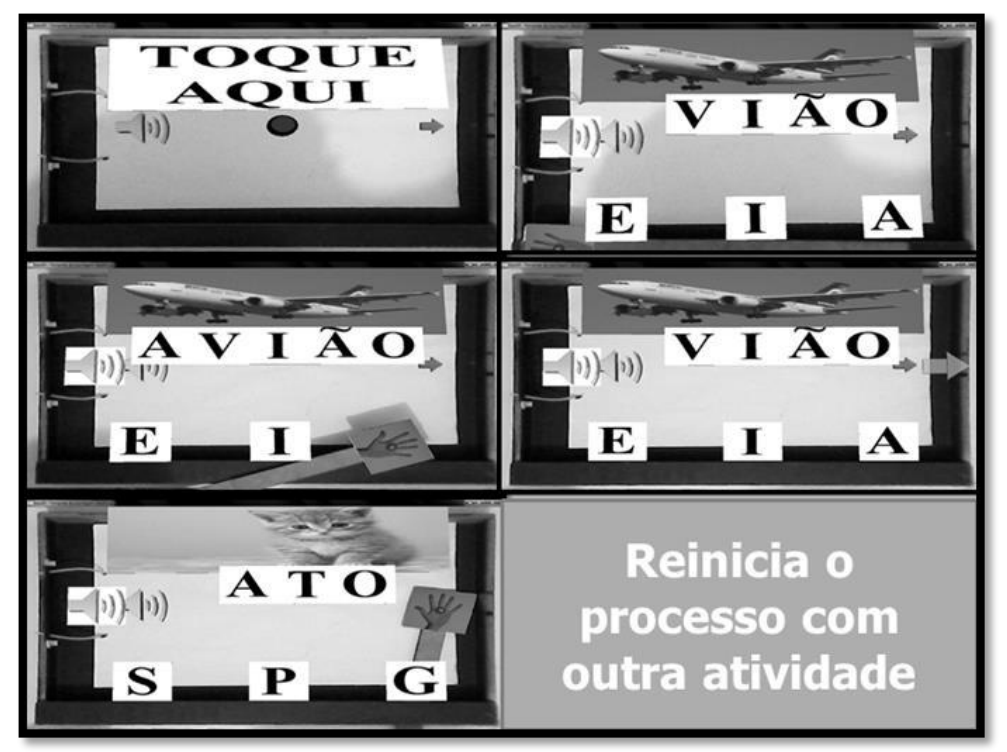

Figura 3. encaixAR em execução.

As atividades do encaixAR envolvem letras e sílabas. Basicamente, o usuário deve escolher a partir de um conjunto de alternativas apresentadas aquela que completa a palavra referente a uma imagem. Por exemplo, na Figura 3 (que ilustra somente as duas primeiras atividades das nove contidas no encaixAR), é possível observar as imagens de um avião e de um gato com os fragmentos de palavra "vião" e "ato", 
respectivamente. Observando a imagem e o fragmento, o usuário deve perceber qual letra está faltando e tocá-la com o marcador atuador.

Quando o usuário conclui todas as atividades, surge um carro 3D descrevendo uma trajetória circular em torno de uma placa de congratulações. O usuário tem a opção de reiniciar, ou interagir com o carro. Esse fim foi proposto como meio de recompensa.

\section{Experimento}

Participaram do experimento 4 professoras e 11 alunos, todos da mesma instituição. As professoras foram responsáveis pela seleção dos alunos, considerando o nível de deficiência e a capacidade de raciocínio do aluno. $\mathrm{O}$ experimento com os alunos foi dividido em três etapas:

- Entrevista: Realizada antes do contato com o encaixAR com a finalidade de conhecer o participante. A entrevista contribui principalmente com a avaliação dos resultados, ou seja, com as informações coletadas na entrevista espera-se observar padrões nos resultados obtidos.

- Momento de interação: A interação do aluno com o encaixAR teve duração média de 30 minutos. Nesse momento, o observador auxiliava os alunos na execução das atividades e anotava as observações sobre o andamento da interação.

- Questionário: Realizado após a interação. Essa etapa coletava dos alunos informações sobre aspectos do encaix $A R$ e a percepção do mesmo sobre a interação.

As Tabelas 1 e 2 apresentam as perguntas feitas aos participantes. A etapa de interação foi realizada em uma sala onde estavam presentes: o professor, o observador e o aluno. Durante esta etapa, eventualmente, a professora ou o observador auxiliavam o aluno na execução de alguma tarefa ou no entendimento das perguntas.

Tabela 1. Entrevista

\begin{tabular}{|l|l|}
\hline NúmeroPergunta \\
\hline $\mathbf{1}$ & Você tem computador em casa? Se sim, como vocế \\
\hline $\mathbf{3}$ & Você gosta do laboratório da escola? \\
\hline $\mathbf{4}$ & Você gosta do que faz na aula de informática? \\
\hline $\mathbf{5}$ & Prefere sala de aula ou informática? \\
\hline & Você já estudou as letras? e as palavras? \\
\hline & TúmeroPela 2. Questionário \\
\hline $\mathbf{1}$ & Você conhecia todas as palavras? \\
\hline $\mathbf{2}$ & Foi fácil entender como usar o livro? \\
\hline $\mathbf{3}$ & Foi fácil usar o livro? \\
\hline $\mathbf{4}$ & Você prefere arrastar ou marcar? \\
\hline $\mathbf{5}$ & Você gostaria de ter aulas com este livro? \\
\hline $\mathbf{6}$ & Você levaria este livro para casa? \\
\hline $\mathbf{7}$ & Você gostou dos sons? \\
\hline $\mathbf{8}$ & Você gostou das figuras? \\
\hline
\end{tabular}


As perguntas, tanto na etapa Entrevista quando na etapa Questionário, foram realizadas oralmente pelo observador com algumas adaptações de forma a facilitar o entendimento do aluno em relação ao que estava sendo questionado.

\subsection{Perfil dos participantes}

Todos os alunos que participaram dos experimentos possuíam alguma deficiência cognitiva e cursavam entre $3^{\mathrm{a}}$ a $4^{\mathrm{a}}$ série do ensino fundamental. A média de idade estava entre 8 e 12 anos para $3^{\mathrm{a}}$ e $4^{\mathrm{a}}$ séries, respectivamente.

Em relação ao perfil do usuário que participou do experimento analisado durante a entrevista, foi possível identificar os seguintes aspectos:

- 46\% dos alunos não possuem computador em casa. Além disso, outros 27\% possuem, mas não usam. Os demais têm acesso livre ao computador em casa e o utilizam somente para jogos. $73 \%$ dos alunos têm contato com o computador exclusivamente na escola. Estes dados enfatizam o papel da escola na inclusão digital.

- Como para a maioria dos alunos a escola é o único meio de acesso ao computador, foi relevante analisar se o ambiente configurado no laboratório durante o experimento e as aulas atendia às expectativas do usuário ou, de alguma forma, causava algum desconforto. Em relação às perguntas 2 e 3 da entrevista (Tabela 1), todos os alunos afirmaram que gostam do laboratório da escola (2) e gostam do que fazem na aula de informática (3). Além disso, alguns alunos descreveram as atividades realizadas na aula de informática demonstrando empolgação.

- De acordo com a Figura 4, 45\% dos alunos não tem preferência por onde as aulas ocorrem. Os 19\% que preferem aulas no laboratório, possuem e utilizam o computador em casa. Já os $36 \%$ que preferem sala de aula estão ou no grupo de alunos que não possuem computador em casa ou possuem e não utilizam. Este resultado foi importante para a condução do experimento de forma a motivar os participantes.

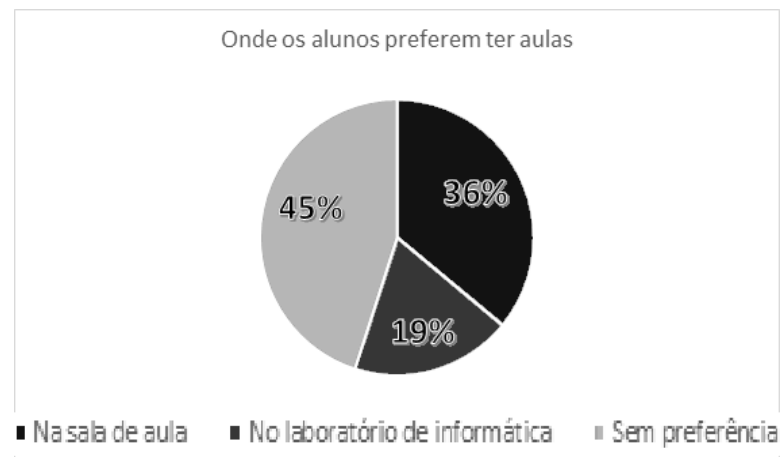

Figura 4. Onde os alunos preferem ter aulas.

- Os alunos envolvidos no experimento possuíam diferentes níveis de conhecimento. Este aspecto poderia interferir diretamente na quantidade de atividades que o mesmo conseguiria realizar. $18 \%$ dos alunos estudaram somente até as letras, $64 \%$ já estudaram as sílabas e $18 \%$ já são capazes de construir frases. 


\section{Resultados}

Após interagirem com a ferramenta as crianças responderam ao questionário. Um dos objetivos foi analisar a experiência de uso. Todos afirmaram ter gostado das figuras e do áudio. Os alunos se divertiram principalmente com o timbre da voz emitida pelo encaixAR (editada para parecer a voz de uma criança).

A satisfação é um ponto importante quando se refere a objetos de aprendizado. Os elementos multimídia contribuem para aumentar a satisfação do usuário. No caso do público em questão, uma maneira de medir a satisfação é avaliar a propensão de utilizar o encaixAR dentro ou fora do ambiente escolar.

Quando perguntados sobre se gostariam de ter as aulas com o encaixAR, 81\% dos alunos afirmaram que gostariam. Além disso, 36\% dos alunos afirmaram que levariam para casa. Os outros $64 \%$ que não levariam o encaixAR para casa disseram que não podem pela indisponibilidade ou inexistência de um computador.

Os alunos, segundo a Figura 5, não encontraram dificuldades durante $o$ experimento. Apenas um aluno foi incapaz de avaliar esse quesito, todos os demais tiveram facilidade em entender o funcionamento e qual era seu papel na interação com o encaixAR.

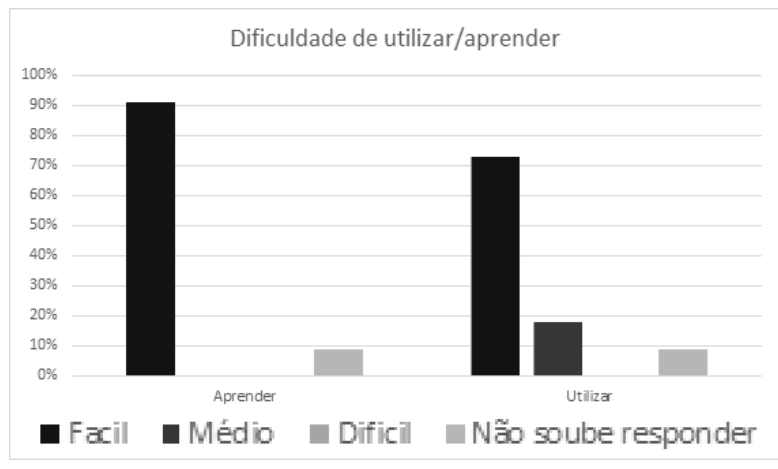

Figura 5. Dificuldade de utilizar/aprender.

Conforme dito anteriormente, o encaixAR possui dois tipos de interação: arrastar ou marcar. Segundo a Figura 6, os alunos preferem as atividades de marcar. O principal problema com as atividades de arrastar é a limitação em relação à coordenação motora. Desta forma, no encaixAR foi possível minimizar os efeitos destas limitações apresentando os dois tipos de atividades.

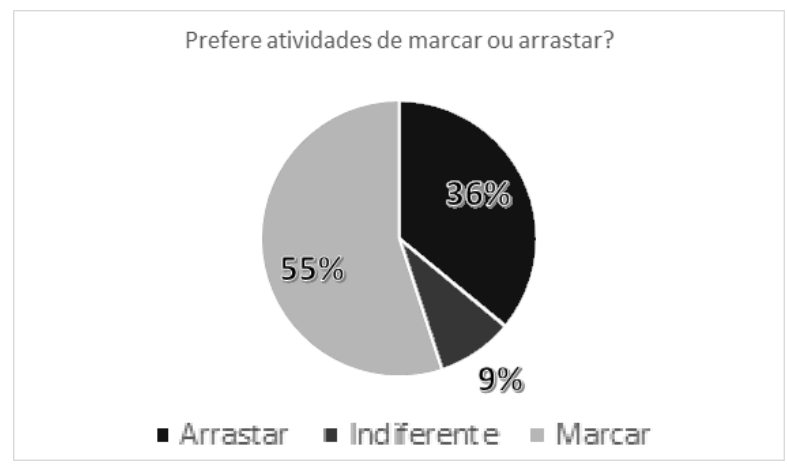

Figura 6. Preferência por funcionalidade. 
Em geral as informações obtidas indicam que a ferramenta encaixAR pode ser aplicada dentro do ambiente escolar alcançando grande parte dos alunos. Parte dessa conclusão vem da observação realizada durante o experimento quando foi possível analisar o comportamento dos alunos.

No quesito autonomia, o encaixAR apresentou bons resultados. $82 \%$ dos alunos precisaram de ajuda no máximo até a $4^{\mathrm{a}}$ atividade e depois executaram todas sem ajuda. Apenas $18 \%$ dos alunos precisaram de ajuda durante a primeira atividade, porém, depois a executaram novamente sem ajuda. Um resultado similar se aplica para a autonomia de troca de atividades, ou seja, $82 \%$ dos alunos entenderam o que deveria ser feito para avançar de atividade no máximo a partir da $3^{\mathrm{a}}$ atividade.

Outros padrões de comportamento dos alunos demonstraram a satisfação provocada pelo encaixAR. Por exemplo, todos os alunos após concluir todas as atividades optaram por recomeçar. Essa repetição acontecia no mínimo 3 vezes, o que sugere que a experiência foi satisfatória.

\section{Conclusões}

Este trabalho apresentou a percepção de crianças PNEE sobre um ambiente com RA, além da adequação do emprego das principais características da RA no desenvolvimento de um aplicativo para o público PNEE.

A principal contribuição desse trabalho foi o ambiente físico adaptado e as estratégias para escolha de multimídia para desenvolver aplicações relacionadas.

O encaixAR foi criado para validar com o público-alvo as opções de implementação e o ambiente físico. Os resultados práticos obtidos vão além do encaixAR propriamente dito. Basicamente, qualquer aplicação em RA que vise o ensino de um determinado conteúdo para PNEE pode utilizar esse trabalho como referência.

Os recursos físicos e virtuais (RA) apresentados nesse trabalho (combinados com a percepção dos usuários PNEE) permitem que novas pesquisas possam focar em aspectos pedagógicos, não analisados neste trabalho. Como trabalho futuro, pretende-se elaborar um experimento para avaliar a eficiência pedagógica da RA para PNEE.

\section{Referências}

Bechtold, P. e Weiss, S. L. I. (2005) "A inclusão das pessoas com necessidades educacionais especiais no mercado de trabalho". Monografia apresentada ao curso de especialização em psicopedagogia do Instituto Catarinense de Pós-graduação. 2005.

Cerqueira, C. S. e Kirner, C. (2011) "basAR: Ferramenta de Autoria de Realidade Aumentada com Comportamento" In: VIII Workshop de realidade virtual e aumentada. Uberaba - MG - 2011.

Dainese, C. A. e Gabin, T. R. (2009) “AmCARA - Ambiente e Comunicação Alternativo com Realidade Aumentada: O acesso do deficiente motor severo a softwares e Web". In: XX Simpósio Brasileiro de Informática na Educação, 2009, Florianópolis - SC. Anais do XX Simpósio Brasileiro de Informática na Educação 2009. Minas Gerais: UFSC, 2009.

Fernandes, F. A, Leite, E. Dos S, Lima, J. V. De, Macedo, S. Da H. e Biasuz, M. C. V. (2012) "Uso de Realidade Aumentada como apoio ao Ensino do Campo Girante de 
um Motor de Corrente Alternada" In: Anais do XXIII Simpósio Brasileiro de Informática na Educação. Rio de Janeiro, 2012.

Kaufmann, H. (2003) "Collaborative Augmented Reality in Education.” In: Imagina Conference 2003 issued by Imagina; Monaco Mediax, Monaco, 2003.

Kirner, C. (2013) "Desenvolvimento de aplicações Educacionais Adaptáveis Online com Realidade Aumentada", In: Tendências e Técnicas em Realidade Aumentada, v. 3, p. 9-25, maio/2013, SBC.

Kirner, C. e Kirner, T. G. (2008) "RVA - Definições", 2008. Disponível em: $<$ http://www.realidadevirtual.com.br/cmsimple-rv/?DEFINI\%C7\%D5ES $>$. Acesso em: 27/09/2013.

Paula, M. M. V. De, Ferreira, G. A. e Silva, R. A. (2012) "Uma análise exploratória do uso da Realidade Aumentada por Pessoas com Necessidades Educativas Especiais", 2012. Anais do XXIII SBIE - Simpósio Brasileiro de Informática na Educação 2012. Rio de Janeiro, RJ, Brasil.

Piske, O. R. (2013) "RUP - Rational Unified Process" 2003 - < disponível em: http://www.angusyoung.org/arquivos/artigos/trabalho_rup.pdf $>$ acessado em: 01/08/2013.

Santin, R. (2008) "SACRA - Sistema de Autoria Em Ambiente Colaborativo com Realidade Aumentada". Piracicaba: UNIMEP - Universidade Metodista de Piracicaba, 2008. 125p. Dissertação - Mestrado em Ciência da Computação, Faculdade de Ciências Exatas e da Natureza.

Souza, F. F. (2010) "Desenvolvimento de Jogos Computacionais como Objetos de Aprendizagem para Pessoas com Necessidades Educativas Especiais”. Dissertação de Mestrado apresentada ao programa de pós-graduação em ciências em ciência e tecnologia da computação - UNIFEI (Universidade Federal de Itajubá) 2010.

Souza, R. C, Moreira H. D. F. e Kirner, C. (2012) "FLARAS 1.0 - Flash Augmented Reality Authoring System", e-book, 2012. Disponível em: $<$ http://ckirner.com/flaras2/wp-content/uploads/2012/09/livro-flaras.pdf $>$. Acesso em: 17/05/2013.

Vaghetti, C. A. O. e Botelho, S. S. Da C. (2010) "Princípios Ergonômicos E Usabilidade de Interface de Realidade Aumentada em Ambientes Virtuais de Aprendizagem: Visão Geral e Tendências", VETOR - Revista de Ciências Exatas e Engenharia, v. 20, n. 1 (2010). 